\title{
Easily adoptable total joint arthroplasty program allows discharge home in two days
}

\section{Un programme facile à adopter d'arthroplastie par prothèse totale permet de donner le congé de l'hôpital après deux jours}

\author{
Michael Raphael • Melanie Jaeger, MD • \\ Janet van Vlymen, MD
}

Received: 8 February 2011/ Accepted: 14 July 2011 / Published online: 6 August 2011

(c) Canadian Anesthesiologists' Society 2011

\begin{abstract}
Purpose A safe efficient care pathway is needed to address the increasing need for arthroplasty surgery in Canada. Our primary objective was to determine whether a fast-track model of care can reduce length of hospital stay following total hip and knee arthroplasty while maintaining patient safety and satisfaction.

Methods In this historical cohort study, 100 patients treated in a newly implemented fast-track program for total joint arthroplasty were compared with 100 patients treated before the introduction of the program. The fast-track program emphasizes preoperative patient education, postoperative multimodal analgesia with periarticular injections, early physiotherapy and rehabilitation, and discharge home with an outpatient rehabilitation program. The primary outcome was hospital length of stay. Secondary outcomes were concerned with patient safety and involved evaluating postoperative side effects, transfers to the tertiary care hospital, and emergency department (ED) visits and readmissions to hospital within 30 days of discharge.
\end{abstract}

Results Length of hospital stay adjusted for age, sex, smoking, comorbidities, American Society of Anesthesiologists' physical status classification, body mass index, and surgical procedure was reduced significantly for patients in

This article is accompanied by an editorial. Please see Can J Anesth 2011; 58(10).

M. Raphael · M. Jaeger, MD · J. van Vlymen, MD ( $\square)$ Department of Anesthesiology and Perioperative Medicine, Kingston General Hospital, Queen's University, 76 Stuart Street, Kingston, ON K7L 2V7, Canada

e-mail: vanvlymj@kgh.kari.net the fast-track program compared with the standard program (mean $47 \mathrm{hr}$; 95\% confidence interval [CI] 41 to 53 vs mean $116 \mathrm{hr}$; 95\% CI 110 to 122, respectively). Patients in the fast-track program were discharged from hospital 69 hr earlier than patients in the standard program $195 \%$ CI -60 to -78). Despite significantly less morphine utilization, pain scores trended lower in the fast-track patients, both at rest and with activity, than in patients in the standard group (median $7.5 \mathrm{vs} 35 \mathrm{mg}$, respectively). There were no significant differences between the two groups in the rate of $E D$ visits or readmissions in the first 30 days.

Conclusion Our multimodal multidisciplinary fast-track protocol reduced hospital stay and opioid consumption while maintaining a high level of patient safety. Program implementation is feasible both in tertiary care and in community hospitals.
Résumé
Objectif Une voie d'accès sécuritaire et efficace aux soins de santé est nécessaire afin de répondre à la demande croissante de chirurgies d'arthroplastie au Canada. Notre objectif principal était de déterminer si un modèle de soins accéléré pouvait réduire la durée de séjour à l'hôpital après une arthroplastie totale de la hanche et du genou tout en maintenant la sécurité et la satisfaction des patients.
Méthode Dans cette étude de cohorte historique, 100 patients traités dans le cadre d'un programme accéléré nouvellement mis en ouvre pour une arthroplastie par prothèse totale ont été comparés à 100 patients traités avant l'introduction du programme. Le programme accéléré met l'emphase sur l'éducation préopératoire du patient, l'analgésie multimodale postopératoire à l'aide d'injections périarticulaires, des séances précoces de physiothérapie et de réadaptation, et un congéde l'hôpital avec un programme 
de réadaptation ambulatoire. Le critère d'évaluation principal était la durée de séjour à l'hôpital. Les critères d'évaluation secondaires touchaient à la sécurité des patients et ont évaluéles effets secondaires postopératoires, les transferts vers un hôpital de soins tertiaires, les visites au département des urgences et les réadmissions à l'hôpital dans les 30 jours suivant le congé.

Résultats La durée de séjour à l'hôpital, telle qu'ajustée pour tenir compte de l'âge, du sexe, du tabagisme, des comorbidités, de la classification de statut physique selon l'American Society of Anesthesiologists, de l'indice de masse corporelle et de l'intervention chirurgicale, était significativement réduite chez les patients dans le programme accéléré par rapport au programme standard (moyenne $47 \mathrm{~h}$; intervalle de confiance $95 \%$ [IC] 41 à $53 \mathrm{vs}$ moyenne 116 h; IC $95 \% 110$ à 122, respectivement). Les patients pris en charge dans le programme accéléré ont reçu leur congé de l'hôpital 69 h plus tôt que les patients pris en charge dans le programme standard (IC $95 \%$-60 à -78). Malgré une utilisation de morphine significativement réduite, les scores de douleur affichaient une tendance plus basse au repos et à l'effort chez les patients du programme accéléré que chez les patients dans le groupe standard (médiane 7.5 vs $35 \mathrm{mg}$, respectivement). Aucune différence significative n'a été observée entre les deux groupes quant au taux de visites à l'urgence ou de réadmission au cours des 30 premiers jours postopératoires.

Conclusion Notre protocole accéléré pluridisciplinaire multimodal a réduit la durée de séjour à l'hôpital et la consommation d'opioïdes tout en maintenant un niveau élevé de sécurité des patients. La mise en ceuvre du programme est faisable dans les hôpitaux de soins tertiaires aussi bien que dans les hôpitaux communautaires.

A fast-track surgery program is a comprehensive guide to the perioperative management of patients in order to achieve clinically and fiscally important outcomes, such as earlier discharge from hospital. ${ }^{1}$ Fast-track models are based on the coordination of multiple individual elements of patient care into an optimized multimodal approach, including targeted patient selection, enhanced patient education, a multimodal approach to postoperative analgesia, and early patient mobilization and physiotherapy. ${ }^{2-4}$

Improvements in surgical technique combined with an evolving understanding of perioperative care have allowed the fast-track concept to be implemented with more complex and invasive surgical procedures. ${ }^{5}$ In orthopedics, this model has been applied successfully to total joint arthroplasty where fast-track programs have been associated with reduced surgical wait times, length of hospital stay, and costs of care. ${ }^{1,4,6-22}$ Importantly, studies suggest that patient safety remains high, and satisfaction is equal or improved. ${ }^{1,4,9,12-16,18,19,21,23}$ However, the resources required for successful implementation of these programs vary greatly in their demand for new technical skills, specialized equipment, trained personnel, and an accommodating geologistic setup. To address the increasing burden on the health care system effectively, a model of care is needed that can be implemented outside of specialty centres with little added investment either in resources or in the mastery of specialized technical skills. In addition, a fast-track program is not suitable for all patients, and success of the program depends on careful screening of appropriate patients. A recent study showed that increasing age, American Society of Anesthesiologists (ASA) physical status, preoperative use of walking aids, low preoperative hemoglobin, and patients living alone were associated with an increased length of stay. ${ }^{10}$

In 2009, we initiated a fast-track comprehensive multidisciplinary lower limb total joint arthroplasty program in our institution. The program was designed as a low-cost easily adoptable fast-track model with a goal to reduce the length of hospital stay at our centre. We developed criteria for enrolment based on our own experience and factors reported in the literature. ${ }^{10}$ Our primary objective was to determine whether a fast-track model of care could reduce length of hospital stay following total hip and knee arthroplasty while maintaining a high level of patient safety and satisfaction. Our secondary objective was to compare the incidence of clinically meaningful outcomes of the fasttrack program with the previous standard joint care program.

\section{Methods}

To establish the safety and efficacy of the newly implemented lower limb total joint fast-track program, we designed a historical cohort study to assess patient outcome before and after the implementation of a fast-track model of care.

\section{Patient selection}

After receiving approval from the Queen's University Health Sciences and Affiliated Teaching Hospitals Research Ethics Board, we abstracted data from the electronic medical records of the first 100 patients in the fasttrack program established in 2009. Patient eligibility for the fast-track program is listed in Table 1. The standard discharge comparison group was then selected from the first 100 patients who underwent arthroplasty from the beginning of 2008 and who would have met the criteria established for the fast-track program listed above. 
Table 1 Fast-track joint program patient eligibility criteria

Patient Criteria

Primary hip or knee replacement

Age $\leq 85$

ASA I-III no cardiac or respiratory functional limitation

BMI $\leq 45$ who are independently mobile

Normal hematocrit

No rheumatoid arthritis

No history of pulmonary embolism or DVT within the last six months

No preoperative coumadin (unless for atrial fibrillation)

Functional strength of upper extremity

Suitable home layout/design (main floor bathroom / bedroom)

Adequate home support (responsible adult to assist the patient at home)

ASA $=$ American Society of Anesthesiologists; $\mathrm{BMI}=$ body mass index; DVT $=$ deep venous thrombosis

An unblinded reviewer abstracted demographic and clinical data from each patient's medical chart which were considered potential confounders of the relationship between the surgical pathway and the primary outcome of interest (length of stay). Demographic and clinical variables included age, sex, body mass index (BMI), smoking status, comorbidities, ASA status, and surgical procedure. The perioperative data collected included duration of surgery, intraoperative anesthetic technique, use of intrathecal morphine and local analgesia, fluid replacement, and estimated blood loss. Patient outcomes of interest were also abstracted, including hospital length of stay, intravenous opioid use, pain scores, postoperative nausea and vomiting (PONV), transfers to the tertiary care hospital, and emergency department (ED) visits or readmission to hospital within 30 days.

Fast-track total joint care plan

The fast-track collaborative care plan was designed as an evidence-based approach to perioperative care of patients by a multidisciplinary team. With initiation of the fasttrack program in April 2009, selected patients underwent their surgery at the university-affiliated ambulatory care facility with an onsite short stay unit (SSU).

The program's action plan was implemented during the patient's surgical consult at the orthopedic clinic as soon as the decision for surgery was made (See the Appendix). Potential candidates were identified, and they were provided with educational material about the procedure, the fast-track program, and the use of low molecular weight heparin (LMWH) for thromboembolic prophylaxis. Discharge planning began at this early stage by reinforcing with the patient and the patient's family an expected discharge on postoperative day (POD) 2. Several weeks prior to surgery, patients attended the pre-surgical clinic for a nursing assessment, preoperative investigations, anesthesiology consultation, and a patient education class.

On the day of surgery, the patient was assessed and given pre-emptive analgesics at the discretion of the attending anesthesiologist. Prior to surgical closure, a periarticular injection was performed by the surgeon. The injection, mixed in a $120 \mathrm{~mL}$ solution, consisted of ropivacaine $300 \mathrm{mg}$ with epinephrine $300 \mu \mathrm{g}, \pm$ morphine 5-10 $\mathrm{mg}$ (if no allergy or sensitivity), and \pm ketorolac $15-30 \mathrm{mg}$ (if there was normal renal function and no other contraindication).

Postoperatively, intravenous patient-controlled opioid analgesia (PCA) was initiated in the postanesthesia care unit (PACU). Patients were transferred to the SSU when they reached standard PACU discharge criteria. Oral coanalgesics were given postoperatively at the discretion of the anesthesiologist. The PCA was discontinued the morning of POD 1 and replaced with oral opioids and nonopioid analgesics. In the SSU, physiotherapy began on POD 0 as soon as the spinal anesthetic had resolved, approximately two to four hours following the surgery. Physiotherapy sessions were employed one to two times on POD 0 , initially emphasizing bed transfers, movement from sitting to standing, and progressing to ambulation 5-10 $\mathrm{m}$ with the assistance of two staff and a walking aid. Patients were instructed to use deep breathing, ankle pumping, static quadriceps, and buttock exercises.

Patients were discharged home when they met the following criteria: pain managed with oral medication; normal vital signs; incision intact with no sign of infection or bleeding; appropriate oral intake without nausea or vomiting; voiding adequately; stable hemoglobin; able to perform self-care, including rehabilitation exercises and injection of LMWH; understands symptoms and signs necessitating return to the hospital; able to carry out activities of daily living with minimal assistance; independent in bed mobility and transfers; ambulating $20 \mathrm{~m}$ with a walking aid; and able to climb stairs with minimal assistance. Patients were transferred to the tertiary care centre under two circumstances: medical emergencies requiring inpatient hospital resources (e.g., other medical consultants) or discharge to home not feasible by POD 4 as the ambulatory care facility does not have weekend coverage. All patients were contacted by the nurse practitioner two to three days after discharge to assess their symptoms and recovery.

Standard elective total joint care program

The fast-track program was initiated at our centre in April 2009. Prior to that date, all total joint patients were 
managed at a university-affiliated tertiary care teaching centre. Following are the key differences of the standard plan (to be discussed below): all surgery was performed at the tertiary care hospital; there was limited preoperative education, no predetermined length of stay plan, minimal discharge planning prior to admission, no standardized preemptive or postoperative multimodal analgesia, use of peripheral nerve blockade for postoperative analgesia, and physiotherapy was initiated on POD 1 if tolerated. Periarticular local anesthetic infiltration was not an analgesic method used during this time period.

Outcome definitions

Hospital length of stay was defined as the time interval from the end of surgery to hospital discharge. Patient reported pain scores, both at rest and with activity, were recorded by the nursing staff every four hours using a numerical rating scale (NRS) of 0-10. An incident of postoperative nausea was defined as any report of nausea recorded by the nurse in the patient records, or in the absence of such a report, any administration of therapeutic antiemetic therapy as indicated by the patient's Medication Administration Record.

\section{Statistics}

Demographic, clinical, and perioperative data are summarized as percents, means, or medians and arranged by surgical pathway. A linear regression model was used to estimate the average difference in time to discharge between surgical pathways while adjusting for potential confounders. Potential confounders included in the analysis were age, BMI, current smoking status, comorbidities, ASA physical status (I/II vs III), and surgical procedure (total hip arthroplasty $v s$ total knee arthroplasty). Statistical significance set at $P<0.05$, and SPSS $\AA$ version 19 (2010, Chicago IL, USA) was used to conduct the analysis. The sample size was chosen based on our view of the appropriate number of patients to enrol in a new program before proceeding with an in-depth review for safety. This was a convenient sample size and not one based on formal sample size calculations.

\section{Results}

The control group of 100 patients in the standard program whose members met the eligibility criteria for the fast-track program (Table 1) was selected after screening 153 consecutive patient charts. Members in the control group were then compared with the first 100 consecutive patients
Table 2 Patient characteristics

\begin{tabular}{lll}
\hline & $\begin{array}{l}\text { Standard Discharge } \\
(n=100)\end{array}$ & $\begin{array}{l}\text { Fast-track Program } \\
(n=100)\end{array}$ \\
\hline $\begin{array}{l}\text { Age, Mean (SD) } \\
\text { Sex, \% }\end{array}$ & $69(8)$ & $65(9)$ \\
Male & 47 & 52 \\
Female & 53 & 48 \\
ASA, \% & & \\
I,II & 59 & 74 \\
III & 41 & 26 \\
BMI, Mean (SD) & $31(5)$ & $29(5)$ \\
Current Smoker, \% & 8 & 18 \\
Comorbidities, \% & & \\
Cardiac Disease & 17 & 14 \\
HTN & 56 & 45 \\
OSA & 9 & 9 \\
COPD & 5 & 2 \\
DM & 16 & 11 \\
Renal Disease & 7 & 10 \\
Surgery, \% & & 57 \\
THA & 31 & 43 \\
TKA & 69 & \\
\hline SD & & \\
\hline
\end{tabular}

$\mathrm{SD}=$ standard deviation; ASA = American Society of Anesthesiologists; $\quad$ BMI = body mass index; $\quad$ HTN = hypertension; OSA = obstructive sleep apnea; COPD = chronic obstructive pulmonary disease; $\mathrm{DM}=$ diabetes mellitus; $\mathrm{THA}=$ total hip arthroplasty; TKA $=$ total knee arthroplasty

recruited into our new fast-track program. The baseline characteristics and aspects of intraoperative care are presented in Tables 2 and 3.

Apparent differences between the groups were noticed regarding the type of surgery, BMI, and ASA physical status (Table 2). More patients in the fast-track group than in the standard program received total hip replacement surgery ( $57 \%$ vs $31 \%$, respectively) and were ASA physical status I or II (74\% vs 59\%, respectively). The majority of procedures in both groups were performed under spinal anesthesia. Patients in the fast-track group received colloid administration more often than patients in the standard program (35 vs 16 times, respectively) (Table 3). In accordance with the difference in analgesic strategy between the fast-track and standard care protocols, almost all of the patients in the fast-track group received a periarticular injection rather than a peripheral nerve block. No patients in the standard group received a periarticular injection (Table 3).

The unadjusted and adjusted differences in mean time to discharge by surgical pathway are presented in Table 4. After adjusting for age, sex, smoking, comorbidities, ASA 
Table 3 Intraoperative data

\begin{tabular}{lll}
\hline & $\begin{array}{l}\text { Standard } \\
\text { Discharge } \\
(n=100)\end{array}$ & $\begin{array}{l}\text { Fast-track } \\
\text { Program } \\
(n=100)\end{array}$ \\
\hline $\begin{array}{l}\text { Surgery time, Mean (SD) } \\
\quad \text { Minutes }\end{array}$ & $88(23)$ & $81(23)$ \\
Anesthetic Technique, \% & & \\
$\quad$ Spinal & 81 & 87 \\
$\quad$ General & 19 & 13 \\
Intrathecal Morphine, \%* & $(n=81)$ & $(n=87)$ \\
$0 \mu \mathrm{g}$ & 15 & 17 \\
$100 \mu \mathrm{g}$ & 81 & 76 \\
$200 \mu \mathrm{g}$ & 3.7 & 6.9 \\
Local Analgesia, \% & & \\
$\quad$ Nil & 47 & 5 \\
$\quad$ Peripheral Nerve Block & 53 & 0 \\
Periarticular Infiltration & 0 & 95 \\
Intraoperative Fluid & & \\
Crystalloid (mL), Mean (SD) & $1,714(475)$ & $1,703(666)$ \\
Colloid, \% & 16 & 35 \\
Total (mL), Mean (SD) & $1,771(467)$ & $1,899(616)$ \\
Intraoperative EBL (mL), Mean (SD) & $312(98)$ & $307(179)$ \\
\hline
\end{tabular}

$\mathrm{SD}=$ standard deviation; $\mathrm{EBL}=$ estimated blood loss; *Rounded to two significant digits

physical status, BMI, and surgical procedure, the patients in the fast-track program were discharged $69 \mathrm{hr}(95 \%$ CI $-60,-78)$ earlier than patients in the standard program (47 vs $116 \mathrm{hr}$, respectively). In the fast-track group, all patients except one were discharged by POD 3 , whereas only eight of the standard discharge patients were discharged by POD 3 (Table 4).

There was a trend towards lower pain scores in the fast-track program compared with the standard program (Figure), even though patients in the fast-track program had lower total patient-controlled analgesia morphine use by POD 1 (median morphine equivalents 7.5 vs $35 \mathrm{mg}$, respectively) (Table 4). Despite this decreased opioid use, the fast-track group had significantly more events of nausea and vomiting on POD 0 and 1 (Table 4). Further analysis within the fast-track group did not reveal an association between intraoperative fluid administration and the incidence of PONV.

Two patients in the fast-track program were transferred to the tertiary care centre for further investigations; one for intraoperative electrocardiogram changes and another with chest pain. Both patients had a cardiology consultation and dobutamine stress echocardiogram. Investigations did not reveal any evidence of coronary ischemia. However, the hospital lengths of stay were increased to three and four days. No significant differences were observed between the fast-track and standard groups in terms of rates of ED visits (14 vs 13, respectively) or readmission within 30 days ( 2 vs 1 , respectively). Readmission diagnoses were postoperative hematoma and infection for the two patients in the fasttrack group and inadequate pain control for the one patient in the standard group.
Table 4 Outcome data

*Adjusted for age, sex, American Society of Anesthesiologists (ASA) physical status, body mass index (BMI), smoking, comorbidities, surgical procedure; $\mathrm{CI}=$ confidence intervals $\mathrm{POD}=$ postoperative day

\begin{tabular}{|c|c|c|c|}
\hline & $\begin{array}{l}\text { Standard } \\
\text { Discharge } \\
(n=100)\end{array}$ & $\begin{array}{l}\text { Fast-track } \\
\text { Program } \\
(n=100)\end{array}$ & $\begin{array}{l}\text { Difference (hr) } \\
\text { (Fast-track - } \\
\text { Standard) }\end{array}$ \\
\hline \multicolumn{4}{|c|}{ Length of Stay (hr), mean $(95 \% \mathrm{CI})$} \\
\hline Unadjusted & 118 (110 to 126$)$ & 45 (43 to 47$)$ & $-73(-64$ to -81$)$ \\
\hline Adjusted* & 116 (110 to 122$)$ & $47(41$ to 53$)$ & $-69(-60$ to -78$)$ \\
\hline \multicolumn{4}{|c|}{ Discharge Day, \% (95\% CI) } \\
\hline POD 0 - POD 2 & $0(\mathrm{n} / \mathrm{a})$ & $93(88$ to 98$)$ & 93 (84 to 97$)$ \\
\hline POD 3 & $8(2.7$ to 13$)$ & $6(1.4$ to 11$)$ & $2.0(-6.3$ to 10$)$ \\
\hline $\mathrm{POD} \geq 4$ & 92 (87 to 97$)$ & $1(-1.0$ to 3.0$)$ & $91(82$ to 95$)$ \\
\hline \multicolumn{4}{|c|}{ Intravenous opioids (mg), median $(95 \% \mathrm{CI})$} \\
\hline Morphine equivalents & $35(24$ to 46$)$ & $7.5(4.4$ to 10$)$ & $-28(-16,-39)$ \\
\hline \multicolumn{4}{|c|}{ Blood Transfusion, \% $(95 \% \mathrm{CI})$} \\
\hline Intraoperative & $0(\mathrm{n} / \mathrm{a})$ & $0(\mathrm{n} / \mathrm{a})$ & $0.0(-4.6$ to 4.6$)$ \\
\hline Postoperative & $8(2.7$ to 13$)$ & $8(2.7$ to 13$)$ & $0.0(-8.7$ to 8.7$)$ \\
\hline \multicolumn{4}{|l|}{ Nausea, \% (95\% CI) } \\
\hline POD 0 & $12(5.7$ to 19$)$ & 46 (36 to 56$)$ & $34(21$ to 45$)$ \\
\hline POD 1 & 19 (11 to 27$)$ & $34(24$ to 43$)$ & $15(1.5$ to 27$)$ \\
\hline \multicolumn{4}{|l|}{ Vomiting, \% (95\% CI) } \\
\hline POD 0 & 7.1 (2.0 to 12$)$ & $36(26$ to 45$)$ & $29(17$ to 40$)$ \\
\hline POD 1 & $12(5.2$ to 18$)$ & $24(16$ to 33$)$ & $12(0.0$ to 23$)$ \\
\hline
\end{tabular}




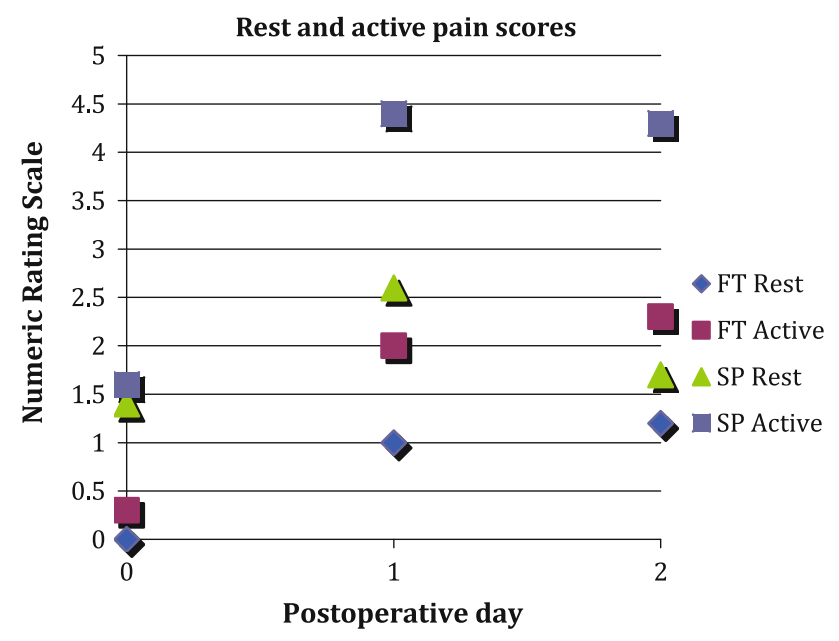

Number of patients for which NRS data available

\begin{tabular}{|l|l|l|l|}
\hline & POD 0 & POD 1 & POD 2 \\
\hline FT Rest & 98 & 84 & 4 \\
\hline FT Active & 95 & 84 & 4 \\
\hline SP Rest & 93 & 87 & 61 \\
\hline SP Active & 60 & 61 & 40 \\
\hline
\end{tabular}

Figure Median rest and active pain scores on a NRS 0-10. $\mathrm{NRS}=$ numeric rating scale; $\mathrm{FT}=$ fast-track; $\mathrm{SP}=$ standard program; $\mathrm{POD}=$ postoperative day

Follow-up phone calls were attempted for all fast-track patients within one week following surgery. Seventy-nine patients were contacted, and all patients reported a good surgical and hospital experience overall. Three patients complained of moderate to severe pain with their oral analgesic (two patients were using low-dose oral opioids, and one patient was using a nonsteroidal anti-inflammatory only). However, none of these patients required a hospital visit on any occasion.

\section{Discussion}

This study has shown that the implementation of an easily adoptable fast-track program can reduce postoperative length of hospital stay while maintaining adequate pain management and safeguarding patient satisfaction and safety. The fast-track program was associated with a shorter length of stay, a trend towards decreased pain scores at rest and with activity throughout the postoperative period, and decreased total intravenous PCA opioid use.
These results were attained without the use of any specialized surgical procedures, additional expensive equipment, or the additional time and expertise required for continuous peripheral nerve block techniques.

The program was designed to emphasize all components involved in lower limb joint arthroplasty equally. The program starts with patient selection; it encompasses education and in-hospital care, and it requires an accommodating environment upon discharge. We did not focus on any single factor, but rather, we concentrated on the entire process as a whole with inclusion of all health care disciplines. The simplicity of the program makes it easy to adopt in any centre. This study was not designed to identify the individual aspects which were most valuable to achieving our outcomes; rather, our purpose was to examine the program as a whole entity.

The concept of multimodal "balanced" analgesia following orthopedic surgery was described over fifteen years ago, ${ }^{24}$ yet it was recognized that pain alleviation, in and of itself, will not result in improvements to surgical outcomes or influence length of hospital stay unless the pain-free period is utilized effectively with rehabilitation. Hebl et al. reported success in decreasing length of stay, improved analgesia, and lower opioid requirements with the introduction of multimodal care pathways. ${ }^{25}$ It was noticed, however, that $48 \%$ of patients were eligible for discharge on POD 2, but discharge was delayed by 1.8 days for reasons of patient disposition. These findings emphasize the need for discharge planning to occur prior to surgery. Many recent studies examining different regimens of stand-alone multimodal analgesia have been able to show important patient outcomes, including reduced postoperative pain, reduced opioid consumption and related sideeffects, and more rapid achievement of functional goals, but they have not shown a decrease in the length of hospital stay. $^{26-28}$

In a recent meta-analysis, it was concluded that the use of regional anesthesia (central neuraxial blockade with spinal or epidural) improves outcomes in patients undergoing arthroplasty of the hip or knee, ${ }^{29}$ although the optimal method of perioperative analgesia has yet to be established. ${ }^{30}$ In several recent studies, comparisons have been made between the benefit of different regimens of local infiltrative analgesia (LIA), epidural analgesia, and peripheral nerve block after orthopedic surgery. ${ }^{31-34} \mathrm{We}$ suggest the use of LIA is well placed in the setting of an easily adoptable fast-track program. Periarticular infiltration is not a technically demanding skill, and it does not result in muscle weakness, which allows rapid initiation of physiotherapy..$^{19,27,28,30,31,33,35-37}$

Our study has several limitations which are important to recognize. The retrospective nature of this study relies both 
on the completeness of the patient chart as well as on the legibility of the handwritten records, not to mention consistency across nurses and institutions with regard to documentation of an event. One of our institutions has incorporated electronic nursing records, whereas the other institution relies on handwritten notes, and this may affect the quality of the information gathered. Perhaps more importantly, we should emphasize that the difference found in hospital length of stay could be due to both recognized and unrecognized differences in our two groups. We accounted for potential recognized differences by adjusting for them statistically. We screened for control patients who would have met the fast-track criteria; however, the screening was done retrospectively, which does not account for the ability of experienced clinicians to identify those patients most likely to succeed in a fast-track program regardless of whether they fit the criteria for inclusion. In addition, we could not retrospectively identify those in the standard program who may have met discharge criteria, but were unable to return home at that time due to an unaccommodating home situation (e.g., available caregiver, ride home, suitable home layout with supports). Lastly, our fast-track program was set up in a facility that was ambulatory in nature. The culture of this facility is different from that of a tertiary care centre managing complex patients with prolonged stays. In this regard, our fast-track facility more resembled a community hospital than a university referral centre. This feature only emphasizes the adoptability of such a program in the general community.

The differences in surgical procedures between the fasttrack and standard groups are likely because inclusion in our fast-track program was limited initially to hip arthroplasty patients only. In our experience, these patients tended to have less postoperative pain than the knee arthroplasty patients. The program was expanded to include total knee arthroplasties once initial success was shown. Subsequently, the number of total hip and total knee patients entered into the fast-track program began to approximate the usual ratio of operations offered at our centre. Other differences between groups, such as ASA physical status, body mass index, and age, were likely due to selection bias inherent in a newly developed program. However, controlling for these baseline differences resulted in only a very small change in the estimated difference between the groups. The difference in surgical operating room time may also reflect this selection bias as well as the differences between institutions, although the absolute difference in surgical time was not considered clinically relevant. Interestingly, despite decreased opioid use, the patients in the fast-track program experienced significantly more events of nausea and vomiting on POD 0 and POD 1. Since there was no difference in known risk factors for PONV in the fast-track group to account for this difference, we hypothesize that it is due either to early mobilization or to differences in event recording between the two institutions.

Future research into the optimization of fast-track programs is needed to answer several key questions. It will be important to identify which components of the program are necessary and most important to achieve positive clinical outcomes. Many authors, including our group, have described the use of preoperative patient education as an important component in their fast-track program. ${ }^{1,15,30}$ However, it has been shown that the use of preoperative education alone before total joint replacement does not improve postoperative pain, functioning, or length of hospital stay. ${ }^{38}$ Nevertheless, we have shown that incorporating appropriate patient selection into a comprehensive fast-track program was a successful strategy in our institution.

In conclusion, our multimodal multidisciplinary fasttrack protocol achieved meaningful reductions in length of hospital stay, opioid consumption, and pain scores while maintaining a high level of patient safety. This program can be easily implemented both in tertiary care centres and in community hospitals.

Acknowledgements This study was funded using departmental research support. The authors sincerely thank Dr. Elizabeth VanDenKerkhof for her tremendous help with the analysis of our data.

The authors also acknowledge the efforts of the following people who were instrumental in the development and success of the FastTrack Total Joint Program: Ms. Tracy Kent-Hillis, Dr. David Pichora, Dr. John Cain, Dr. John Rudan, Dr. Gavin Wood, Dr. Mark Harrison, Dr. Aaron Campbell, Dr. Davide Bardana, and all members of the Ambulatory Total Joint Committee.

Conflicts of interest None declared. 


\section{Appendix: Summary of the Key Elements of the Fast-track Discharge Protocol}

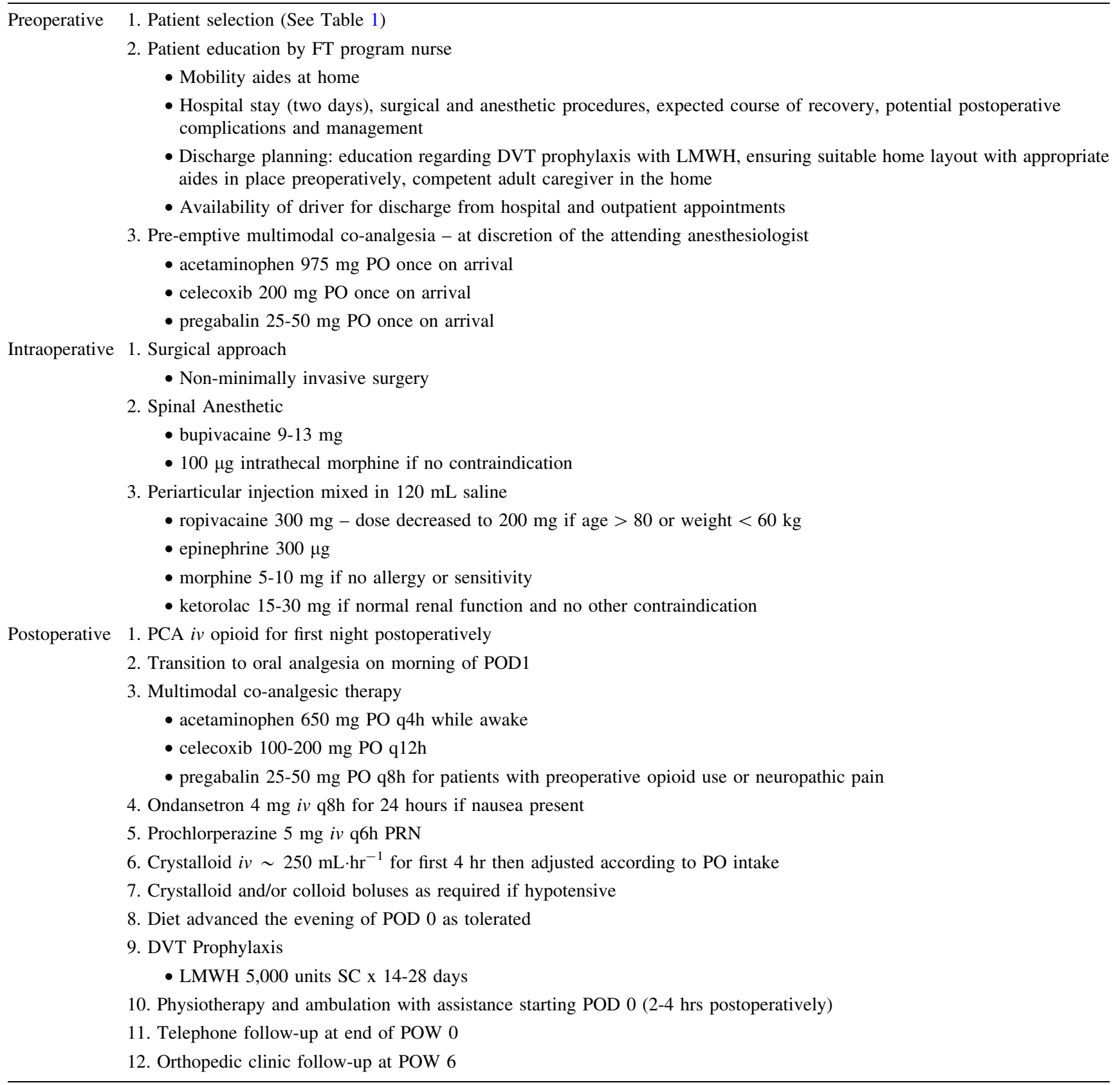

FT = fast-track; PT = physiotherapy; OT = occupational therapy; PO = per os; POD = postoperative day; DVT = deep vein thrombosis; LMWH $=$ low molecular weight heparin; $i v=$ intravenous; PRN $=$ as needed; POW $=$ postoperative week; PCA = patient controlled analgesia; $\mathrm{SC}=$ subcutaneously

\section{References}

1. Larsen K, Hvass KE, Hansen TB, Thomsen PB, Soballe $K$. Effectiveness of accelerated perioperative care and rehabilitation intervention compared to current intervention after hip and knee arthroplasty. A before-after trial of 247 patients with a 3-month follow-up. BMC Musculoskelet Disord 2008; 9: 59.

2. Kehlet H, Wilmore DW. Multimodal strategies to improve surgical outcome. Am J Surg 2002; 183: 630-41. 
3. Kehlet H, Dahl JB. Anaesthesia, surgery, and challenges in postoperative recovery. Lancet 2003; 362: 1921-8.

4. Wainwright T, Middleton $R$. An orthopaedic enhanced recovery pathway. Curr Anaesth Crit Care 2010; 21: 114-20.

5. Kehlet H, Wilmore DW. Evidence-based surgical care and the evolution of fast-track surgery. Ann Surg 2008; 248: 189-98.

6. Brunenberg DE, van Steyn MJ, Sluimer JC, Bekebrede LL, Bulstra $S K$, Joore MA. Joint recovery programme versus usual care: an economic evaluation of a clinical pathway for joint replacement surgery. Med Care 2005; 43: 1018-26.

7. Larsen $K$, Hansen $T B$, Thomsen PB, Christiansen $T$, Soballe $K$. Cost-effectiveness of accelerated perioperative care and rehabilitation after total hip and knee arthroplasty. J Bone Joint Surg Am 2009; 91: 761-72.

8. Andersen LO, Gaarn-Larsen L, Kristensen BB, Husted H, Otte $K S$, Kehlet $H$. Subacute pain and function after fast-track hip and knee arthroplasty. Anaesthesia 2009; 64: 508-13.

9. Husted H, Otte KS, Kristensen BB, Orsnes T, Kehlet H. Readmissions after fast-track hip and knee arthroplasty. Arch Orthop Trauma Surg 2010; 130: 1185-91.

10. Husted H, Holm G, Jacobsen S. Predictors of length of stay and patient satisfaction after hip and knee replacement surgery: fasttrack experience in 712 patients. Acta Orthop 2008; 79: 168-73.

11. Berger RA, Sanders SA, Thill ES, Sporer SM, Della Valle C. Newer anesthesia and rehabilitation protocols enable outpatient hip replacement in selected patients. Clin Orthop Relat Res 2009; 467: 1424-30.

12. Larsen $K$, Sorensen $O G$, Hansen TB, Thomsen PB, Soballe $K$. Accelerated perioperative care and rehabilitation intervention for hip and knee replacement is effective: a randomized clinical trial involving 87 patients with 3 months of follow-up. Acta Orthop 2008; 79: 149-59.

13. Petersen MK, Madsen C, Andersen NT, Soballe K. Efficacy of multimodal optimization of mobilization and nutrition in patients undergoing hip replacement: a randomized clinical trial. Acta Anaesthesiol Scand 2006; 50: 712-7.

14. Peters $C L$, Shirley B, Erickson $J$. The effect of a new multimodal perioperative anesthetic regimen on postoperative pain, side effects, rehabilitation, and length of hospital stay after total joint arthroplasty. J Arthroplasty 2006; 21: 132-8.

15. Ayalon $O$, Liu S, Flies S, Cahill J, Juliano K, Cornell CN. A multimodal clinical pathway can reduce length of stay after total knee arthroplasty. HSSJ 2011; 7: 9-15.

16. Cook JR, Warren M, Ganley KJ, Prefontaine P, Wylie JW. A comprehensive joint replacement program for total knee arthroplasty: a descriptive study. BMC Musculoskelet Disord 2008; 9: 154.

17. Duellman TJ, Gaffigan C, Milbrandt JC, Allan DG. Multi-modal, pre-emptive analgesia decreases the length of hospital stay following total joint arthroplasty. Orthopedics 2009; 32: 167.

18. Khan F, Ng L, Gonzalez S, Hale T, Turner-Stokes L. Multidisciplinary rehabilitation programmes following joint replacement at the hip and knee in chronic arthropathy. Cochrane Database Syst Rev 2008; 2: CD004957.

19. Parvataneni HK, Shah VP, Howard H, Cole N, Ranawat AS, Ranawat CS. Controlling pain after total hip and knee arthroplasty using a multimodal protocol with local periarticular injections: a prospective randomized study. J Arthroplasty 2007; 22: 33-8.

20. Bottros J, Klika AK, Milidonis MK, Toetz, A, Fehribach A, Barsoum $W K$. A rapid recovery program after total hip arthroplasty. Curr Orthopaed Pract 2010; 21: 381-4.

21. Dowsey MM, Kilgour ML, Santamaria NM, Choong PF. Clinical pathways in hip and knee arthroplasty: a prospective randomised controlled study. Med J Aust 1999; 170: 59-62.
22. Duncan CM, Hall Long K, Warner DO, Hebl JR. The economic implications of a multimodal analgesic regimen for patients undergoing major orthopedic surgery: a comparative study of direct costs. Reg Anesth Pain Med 2009; 34: 301-7.

23. Hayes JH, Cleary R, Gillespie WJ, Pinder IM, Sher JL. Are clinical and patient assessed outcomes affected by reducing length of hospital stay for total hip arthroplasty? J Arthroplasty 2000; 15: 448-52.

24. Moiniche S, Hjortso NC, Hansen BL, et al. The effect of balanced analgesia on early convalescence after major orthopaedic surgery. Acta Anaesthesiol Scand 1994; 38: 328-35.

25. Hebl JR, Dilger JA, Byer DE, et al. A pre-emptive multimodal pathway featuring peripheral nerve block improves perioperative outcomes after major orthopedic surgery. Reg Anesth Pain Med 2008; 33: 510-7.

26. Lee KJ, Min BW, Bae KC, Cho $C H$, Kwon DH. Efficacy of multimodal pain control protocol in the setting of total hip arthroplasty. Clin Orthop Surg 2009; 1: 155-60.

27. Vendittoli PA, Makinen P, Drolet $P$, et al. A multimodal analgesia protocol for total knee arthroplasty. A randomized, controlled study. J Bone Joint Surg Am 2006; 88: 282-9.

28. Busch CA, Shore BJ, Bhandari R, et al. Efficacy of periarticular multimodal drug injection in total knee arthroplasty. A randomized trial. J Bone Joint Surg Am 2006; 88: 959-63.

29. Hu S, Zhang ZY, Hua YQ, Li J, Cai ZD. A comparison of regional and general anaesthesia for total replacement of the hip or knee: a meta-analysis. J Bone Joint Surg Br 2009; 91: 935-42.

30. Maheshwari AV, Blum YC, Shekhar L, Ranawat AS, Ranawat CS. Multimodal pain management after total hip and knee arthroplasty at the Ranawat Orthopaedic Center. Clin Orthop Relat Res 2009; 467: 1418-23.

31. Spreng UJ, Dahl V, Hjall A, Fagerland MW, Raeder J. Highvolume local infiltration analgesia combined with intravenous or local ketorolac + morphine compared with epidural analgesia after total knee arthroplasty. Br J Anaesth 2010; 105: 675-82.

32. Carli F, Clemente A, Asenjo JF, et al. Analgesia and functional outcome after total knee arthroplasty: periarticular infiltration vs continuous femoral nerve block. Br J Anaesth 2010; 105: 185-95.

33. Toftdahl K, Nikolajsen L, Haraldsted V, Madsen F, Tonnesen EK, Soballe $K$. Comparison of peri- and intraarticular analgesia with femoral nerve block after total knee arthroplasty: a randomized clinical trial. Acta Orthop 2007; 78: 172-9.

34. Fowler SJ, Symons J, Sabato S, Myles PS. Epidural analgesia compared with peripheral nerve blockade after major knee surgery: a systematic review and meta-analysis of randomized trials. Br J Anaesth 2008; 100: 154-64.

35. Busch CA, Whitehouse MR, Shore BJ, MacDonald SJ, McCalden $R W$, Bourne RB. The efficacy of periarticular multimodal drug infiltration in total hip arthroplasty. Clin Orthop Relat Res 2010; 468: 2152-9.

36. Kerr DR, Kohan L. Local infiltration analgesia: a technique for the control of acute postoperative pain following knee and hip surgery: a case study of 325 patients. Acta Orthop 2008; 79: 17483.

37. Rostlund $T$, Kehlet $H$. High-dose local infiltration analgesia after hip and knee replacement-what is it, why does it work, and what are the future challenges? Acta Orthop 2007; 78: 159-61.

38. McDonald S, Hetrick S, Green S. Pre-operative education for hip or knee replacement. Cochrane Database Syst Rev 2004; 1: CD003526. 